\title{
Certain Aspects of Mittag-Leffler Functions in Kober Operator
}

\author{
Sethukumarasamy K, Ambrose Prabhu R, Vijayaraju P, Bhaskaran S
}

\begin{abstract}
Motivated by the triumph of the applications of Mittag-Leffler functions in various fields of engineering sciences, we present the effect ofg Kober operator and extended Kober operator of first kind on the Mittag-Leffler function with three parameters $E_{\alpha, \beta}^{\gamma}(z)$. An effort has been made to investigate the properties of $E_{\alpha, \beta}^{\gamma}(z)$ due to application of Kober fractional integral operator of the first kind of order $\alpha$ We also provide certain results pertaining to the special cases of in the extended Kober operator of first kind.
\end{abstract}

Keywords: Primary 33E12, 26A33 Mittag-Leffler function, Kober operator, Fractional integral operator

\section{INTRODUCTION}

The special function of the form

$$
E_{\rho}(z)=\sum_{k=0}^{\infty} \frac{z^{k}}{\Gamma(\rho k+1)}, \text { for } \rho \in \mathbb{C}, \mathfrak{R}(\rho)>0
$$

and more general function

$$
E_{\rho, \mu}(z)=\sum_{k=0}^{\infty} \frac{z^{k}}{\Gamma(\rho k+\mu)}, \text { for } \rho, \mu \in \mathbb{C}, \Re(\rho)>0
$$

with $\mathbb{C}$ being the set of complex numbers, are known as Mittag-Leffler functions. The function defined in equation (1) was introduced by Mittag-Leffler [13], whereas the function in equation (2) was defined by Wiman [20]. Prabhakar [15] introduced a function $E_{\rho, \mu}^{\gamma}(z)$ of the form

$$
E_{\rho, \mu}^{\gamma}(z)=\sum_{k=0}^{\infty} \frac{(\gamma)_{k}}{\Gamma(\rho k+\mu)} \frac{z^{k}}{k !}, \rho, \mu, \gamma \in \mathbb{C}, \Re(\rho)>0
$$

where $(\gamma)_{k}$ is the Pochhammer symbol defined as

$$
(\gamma)_{k}=(\gamma)(\gamma+1) \cdots(\gamma+k-1), \quad \text { and }(\gamma)_{0}=1 .
$$

The extended Mittag-Leffler function is defined by Özarslan et al. [14] as

$$
\begin{aligned}
& E_{\alpha, \beta}^{(\gamma ; c)}(z ; p)=\sum_{k=0}^{\infty} \frac{B_{p}(\gamma+k, c-\gamma)}{B(\gamma, c-\gamma)} \frac{(c)_{k}}{\Gamma(\alpha k+\beta)} \frac{z^{k}}{k !}, \\
& \text { where } \mathfrak{R}(c)>\Re(\gamma)>0, \text { also } \\
& B_{p}(x, y)=\int_{0}^{1} t^{x-1}(1-t)^{y-1} \exp \left\{-\frac{p}{t(1-t)}\right\} d t \\
& \text { in which }(\mathfrak{R}(p)>0, \mathfrak{R}(x)>0, \mathfrak{R}(y)>0) .
\end{aligned}
$$

When $p=0$ the function $B_{p}(x, y)$ becomes an ordinary beta function

$$
B(x, y)=\int_{0}^{1} t^{x-1}(1-t)^{y-1} d t
$$

Recently, Ramachandran and Ambrose Prabhu[16] revealed the radius of starlikeness of certain order of integral operator and Vijayaraju et al., [18] studied the radius of starlikeness of certain order of a fractional integral operator

Revised Manuscript Received on August 05, 2019.

Sethukumarasamy K, Department of Mathematics, Anna University, Chennai, Tamilnadu, India (Email: mathsethu@gmail.com)

Ambrose Prabhu R, Department of Science and Humanities, Saveetha School of Engineering,Chennai-602105, Tamilnadu, India (Email: ancyamb@yahoo.com

Vijayaraju P, Department of Mathematics, Anna University, Chennai, Tamilnadu, India (Email: vijay@annauniv.edu)

Bhaskaran S, Department of Science and Humanities,Saveetha School of Engineering, Chennai-602105, Tamilnadu, India.(Email: bhaskaran.hawk@gmail.com) related to Erdélyi-Kober operator. In this article, certain properties of Mittag-Leffler function associated with Kober fractional integral operator has been studied. To prove the main results, the following preliminaries are required.

\section{PRELIMINARIES}

The fractional ordered integral is the generalization of $n$-fold integral and fractional derivative means the derivative of arbitrary order, where $n$ not necessarily an nonnegative integer. Earlier the concept of fractional integral and derivatives were purely theoretical and useful only for mathematicians.

In recent days fractional derivatives and fractional integrals are applied in most of the branches of mathematics such as calculus of variations [1,2,5], differential equations $[10,19]$ and numerical analysis [3, 4, 12]. Furthermore, there are various researchers in the field pointed out that integrals and derivatives of non-integer order are very suitable for the description of various applications such as physical, biological, engineering and pure sciences. In fact, differential equations of arbitrary order have applications in various fields. For example, the study of viscoelastic materials which have an intermediate behavior of viscosity and elasticity. This kind of materials are studied by fractional diffusion equation [9].

The exponential function $e^{z}$ plays a vital role in the theory of ordinary differential equations. Similarly, twoparameter Mittag-Leffler function given by equation (2) is quite significant in the theory of fractional differential equations [7].

There are more than one definitions of fractional derivatives available in the literature such as RiemannLiouville fractional derivative, Gruwald-Letnikov fractional derivative and Caputo fractional derivative [4]. Here, we deal with Riemann Liouville fractional integral and Kober Integral operators only.

Definition 1 [17] The left-sided Riemann-Liouville fractional integral of order $\alpha$ is defined as follows:

$$
{ }_{a} I_{x}^{\alpha} f(x)={ }_{a} D_{x}^{-\alpha} f(x)=
$$

$\frac{1}{\Gamma(\alpha)} \int_{a}^{x}(x-t)^{\alpha-1} f(t) d t$, for $\Re(\alpha)>0$

An operator of fractional integration generalizing equation (6) through association of power weight was introduced by Kober [8] in 1940. 
Definition 2 [11] The Kober fractional integral operator of the first kind of order $\alpha$ is defined and denoted as follows:

$I_{x}^{\eta, \alpha}=K_{1, x, \eta}^{-\alpha} f(x)=$

$\frac{x^{-\eta-\alpha}}{\Gamma(\alpha)} \int_{0}^{x}(x-t)^{\alpha-1} t^{\eta} f(t) d t$, for $\Re(\alpha)>0$

Definition 3 [11] The extended Kober operator of the first kind is defined by,

$$
\begin{aligned}
& I_{x+}^{\eta, \alpha} f(x)= \\
& \frac{2 x^{-2 \eta-2 \alpha}}{\Gamma(\alpha)} \int_{0}^{x}\left(x^{2}-t^{2}\right)^{\alpha-1} t^{2 \eta+1} f(t) d t, \text { for } \Re(\alpha)>0
\end{aligned}
$$

\section{MAIN RESULTS}

\subsection{Kober Operator and Mittag-Leffler Function}

Theorem 4 If $\mathfrak{R}(\alpha)>0$ and $\mathfrak{R}(\rho)>0$ then

$$
K_{1, x, \eta}^{-\alpha} E_{\rho, \mu}^{\gamma}(x)=x^{-\eta} \sum_{k=0}^{\infty} \frac{(\gamma)_{k} \Gamma(\eta+k+1)}{\Gamma(\rho k+\mu) k ! \Gamma(\eta+k+\alpha+1)} \text {. }
$$

Proof. By applying the Kober integral operator on the Prabhakar function,

$$
\begin{aligned}
& K_{1, x, \eta}^{-\alpha} E_{\rho, \mu}^{\gamma}(x)=\frac{x^{-\eta-\alpha}}{\Gamma(\alpha)} \int_{0}^{x}(x \\
& -t)^{\alpha-1} t^{\eta} \sum_{k=0}^{\infty} \frac{(\gamma)_{k}}{\Gamma(\rho k+\mu)} \frac{t^{k}}{k !} d t \\
& =\frac{x^{-\eta-\alpha}}{\Gamma(\alpha)} \sum_{k=0}^{\infty} \frac{(\gamma)_{k}}{\Gamma(\rho k+\mu) k !} \int_{0}^{x}(x-t)^{\alpha-1} t^{\eta+k} d t .
\end{aligned}
$$

By introducing simple substitutions, the above equation reduces to

$$
\begin{gathered}
K_{1, x, \eta}^{-\alpha} E_{\rho, \mu}^{\gamma}(x)=\frac{x^{-\eta-\alpha}}{\Gamma(\alpha)} \sum_{k=0}^{\infty} \frac{(\gamma)_{k}}{\Gamma(\delta k+1) k !} \int_{0}^{1}(x(1 \\
=\frac{x^{-\eta}}{\Gamma(\alpha)} \sum_{k=0}^{\infty} \frac{(\gamma)_{k}}{\Gamma(\rho k+\mu) k !} B(\eta+k+1, \alpha) .
\end{gathered}
$$

Now, by applying the property of beta function, the required result is obtained.

Corollary 5 If $\mathfrak{R}(\alpha)>0$ and $\mathfrak{R}(\delta)>-1, \gamma=1$ and $\mu=1$ in Theorem 4, then

$$
K_{1, x, \eta}^{-\alpha} E_{\rho}(x)=x^{-\eta} \sum_{k=0}^{\infty} \frac{\Gamma(\eta+k+1)}{\Gamma(\rho k+1) \Gamma(\eta+k+\alpha+1)} .
$$

3.2 Extended Kober Operator of First Kind and MittagLeffler Function

$$
\begin{aligned}
& \text { Theorem } 6 \text { If } \mathfrak{R}(\alpha)>0 \text { and } \mathfrak{R}(\rho)>0 \text {, then } \\
& \qquad I_{x+}^{\eta, \alpha} E_{\rho, \mu}^{\gamma}(x)=\sum_{k=0}^{\infty} \frac{(\gamma)_{k} \Gamma\left(\eta+\frac{k}{2}+1\right)}{\Gamma(\rho k+\mu) k ! \Gamma\left(\eta+\frac{k}{2}+1+\alpha\right)} .
\end{aligned}
$$

A straightforward application of extended Kober operator on Prabhakar function gives,

Proof.

$$
\begin{gathered}
I_{x+}^{\eta, \alpha} E_{\rho, \mu}^{\gamma}(x)=\frac{2 x^{-2 \eta-2 \alpha}}{\Gamma(\alpha)} \int_{0}^{x}\left(x^{2}\right. \\
\left.\quad-t^{2}\right)^{\alpha-1} t^{2 \eta+1} \sum_{k=0}^{\infty} \frac{(\gamma)_{k}}{\Gamma(\rho k+\mu)} \frac{t^{k}}{k !} d t \\
=\frac{2 x^{-2(\eta+\alpha)}}{\Gamma(\alpha)} \sum_{k=0}^{\infty} \frac{(\gamma)_{k}}{\Gamma(\rho k+\mu) k !} \int_{0}^{x}\left(x^{2}-t^{2}\right)^{\alpha-1} t^{2 \eta+k+1} d t .
\end{gathered}
$$

By taking a simple substitution for $t$ the above equation becomes

$$
\begin{gathered}
I_{x+}^{\eta, \alpha} E_{\rho, \mu}^{\gamma}(x)=\frac{2 x^{-2(\eta+\alpha)}}{\Gamma(\alpha)} \sum_{k=0}^{\infty} \frac{(\gamma)_{k}}{\Gamma(\rho k+\mu) k !} \int_{0}^{1}\left(x^{2}(1\right. \\
-s))^{\alpha-1}\left(s^{\frac{1}{2}} x\right)^{2 \eta+k+1} \frac{x}{2 s^{\frac{1}{2}}} d s \\
=\frac{1}{\Gamma(\alpha)} \sum_{k=0}^{\infty} \frac{(\gamma)_{k}}{\Gamma(\rho k+\mu) k !} B\left(\eta+\frac{k}{2}+1, \alpha\right) .
\end{gathered}
$$

By applying the property of beta function the required result is obtained.

Corollary 7 If $\mathfrak{R}(\alpha)>0$ and $\mathfrak{R}(\rho)>-1, \gamma=1$ and $\mu=1$ in Theorem 6, then

$$
I_{x+}^{\eta, \alpha} E_{\rho}(x)=\sum_{k=0}^{\infty} \frac{x^{k} \Gamma\left(\eta+\frac{k}{2}+1\right)}{\Gamma(\rho k+1) \Gamma\left(\eta+\frac{k}{2}+1+\alpha\right)} .
$$

\section{REFERENCES}

1. Agrawal, O., Fractional variational calculus and the transversality conditions, J. Phys. A 39, 10375-10384, (2006).

2. Almeida, R., Torres, D., Necessary and suffcient conditions for the fractional calculus of variations with Caputo derivatives, Commun. Nonlinear Sci. Numer Simul. 16(3), 1490-1500, (2011).

3. Chen, W., Ye, K., Sun, H, Fractional diffusion equations by the Kansa Method. Comput. Math. Appl. 59, 16141620, (2010)

4. Diethelm, K., the Analysis of Fractional Differential Equations, Springer, Heidelberg (2004).

5. Ferreira R., Torres, D., Isoperimetricproblems of the calculus of variations on time scales, Nonlinear Anal Optim. II Contemp. Math. 514, 123-131, (2010).

6. Gorenflo, R., Kilbas, A. A., Mainardi, F., Rogosin, S.V., Mittag-Leffler Functions, Related Topics and Applications. Ser. Springer Monographs in Mathematics, Springer, (2014).

7. Podlubny, I., Fractional Differential Equations, Academic Press, San Diego, CA, (1999).

8. Kober, H., On a fractional integral and derivative, Quart J. Math. Oxford, 11, 193-211, (1940).

9. Luchko, Y., Initial-boundary-value problems for the onedimensional time-fractional diffusion equation, Fract Calc. Appl. Anal. 15, 141-160, (2012).

10. Masaeva, O., Dirichlet problem for the generalized Laplace equation with the Caputo derivative, Differ. Equ., 48(3), 449-454, (2012).

11. Mathai, A.M., Saxena R.K., Hans J. Haubold, H Function: Theory and Application, Springer, (2009).

12. Meerschaert, M., Tadjeran, C., Finite difference approximation for fractional advection-dispersion flow equations, J. Comput. Appl. Math. 172, 65-77, (2004).

13. Mittag-Leffler,G.M., Sur la nouvelle fonction $E_{\alpha}(x)$. C.R. Acad. Sci. Paris, 137, 554-558 (1903).

14. Özarslan, M.A. Yılmaz, B. The extended Mittag-Leffler function and its properties, J. Inequal. Appl. 85, 1-10, (2014).

15. Prabhakar, T. R., A singular integral equation with a generalized Mittag-Leffler function in the kernel, Yokohama. Math. J., 19, 7-15, (1971). 
16. Ramachandran,C., Ambrose Prabhu, R., Certain properties of analytic functions with an integral operator, Global J. Pure Appl. Math. 12, 16-18, (2016).

17. Samko, S. G., Kilbas, A. A., and Marichev, O. I., Fractional Integrals and Derivatives: Theory and Applications., Gordon and Breach, Yverdon et alibi, (1993).

18. Vijayaraju, R., Sethukumarasamy K., Ambrose Prabhu, R., Certain aspects of analyticity in view of integral operator, Global J. Pure Appl. Math. 114, 37-42, (2017).

19. Wang, J., Lv, L., Zhou, Y., Boundary value problems for fractional differential equations involving Caputo derivative in Banach spaces, J. Appl. Math. Comput. 38, 209-224 (2012).

20. Wiman, A., Über den Fundamental satz in der Theorie der Functionen $E_{\alpha}(x)$. Acta Math., 29, 191-201 (1905). 